\title{
Intracellular rod-like crystals in chronic lymphocyte leukemia
}

\author{
Yiling Huang ${ }^{1} \cdot$ Lixia Zhang $^{1}$ (1)
}

Received: 19 October 2019 / Revised: 26 February 2020 / Accepted: 2 July 2020 / Published online: 14 July 2020

(c) Japanese Society of Hematology 2020

A 78-year-old man was referred to our hospital for acute intestinal obstruction. A CT scan of the abdomen showed multiple lymph nodes in the abdomen and groin. CBC showed: leukocytosis $\left(24.83 \times 10^{9} / \mathrm{L}\right)$ with $61.4 \%$ lymphocyte, hemoglobin concentration $144 \mathrm{~g} / \mathrm{L}$ and platelet count $150 \times 10^{9} / \mathrm{L}$. The peripheral blood smear showed mature lymphocyte with visible one to five rod-like crystals (Fig. 1a-d). Flow cytometry immunophenotyping confirmed these are monoclonal B-cells (42\%), expressed CD45, CD19, CD20, CD22, CD79b, CD5, CD23, CD148 and CD200(hi), partial expressed lambda, and negative for FMC7, CD10, CD11c, CD49d, CD103, CD38, CD25, CD160, IgM, CD81, kappa and Ki67. Chronic lymphocyte leukemia (CLL) is finally diagnosed.

Intracellular rod-like crystal is extremely rarely described in the peripheral blood. Peters et al. [1] reported this anomaly in B cell lymphoproliferative diseases. The inclusions are thought to be abnormal immunoglobulins, it could be hypothesized that lambda light chain possesses such properties, which are precipitated in the cytoplasm.

While rare cases of B cell lymphoproliferative diseases with rod-like crystals inclusions have previously been reported, we believe the phenomenon is not well known, even among experienced hematologists and hematopathologists. Such findings can assist in diagnosis of B cell lymphoproliferative diseases.

Lixia Zhang

Zhanglixia7602@jsph.org.cn

1 Department of Laboratory Medicine, The First Affiliated Hospital of Nanjing Medical University, Nanjing 210029, People's Republic of China

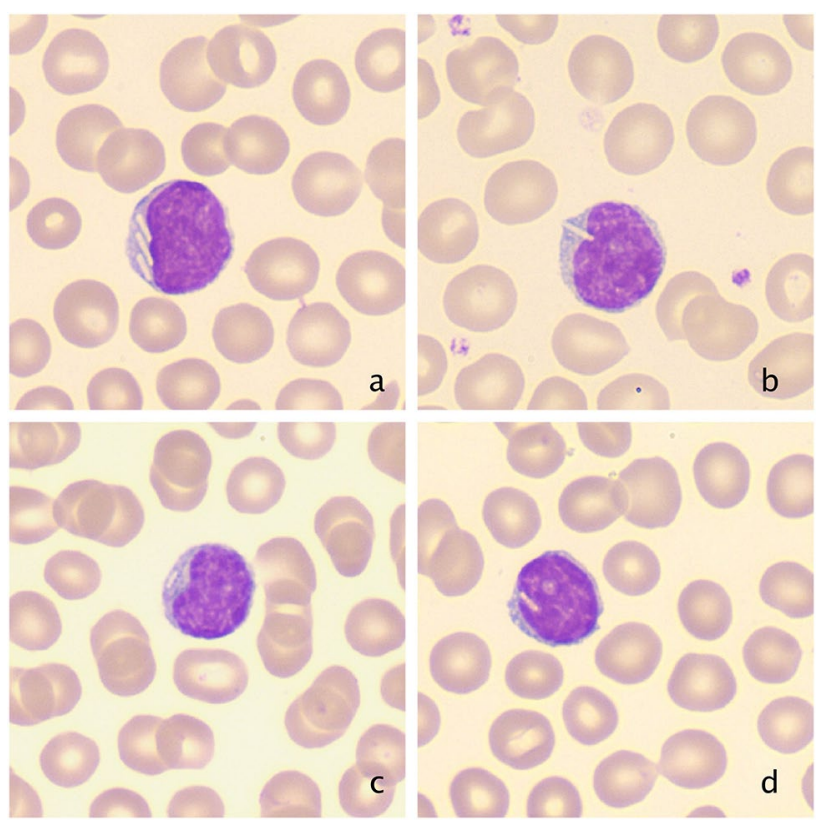

Fig. 1 Visible rod-like crystals presented in the mature lymphocytes $(\times 1000$, Wright Giemsa). a three rod-like crystals. b-d one rod-like crystal

\section{Compliance with ethical standards}

Conflict of interest The authors do not have any conflict of interest to disclosure.

\section{Reference}

1. Peters O, Thielemans C, Steenssens L, De Waele M, Hijmans W, Van Camp B. Intracellular inclusion bodies in 14 patients with B cell lymphoproliferative disorders. J Clin Pathol. 1984;37(1):45-50.

Publisher's Note Springer Nature remains neutral with regard to jurisdictional claims in published maps and institutional affiliations. 